\title{
Assessing the robustness of the networks of spatial genetic variation $^{\dagger}$
}

Eva M. Albert, ${ }^{1}$ Miguel A. Fortuna, ${ }^{1}$ José A. Godoy ${ }^{1}$ and Jordi Bascompte ${ }^{1 *}$

\begin{abstract}
Habitat transformation is one of the leading drivers of biodiversity loss. The ecological effects of this transformation have mainly been addressed at the demographic level, for example, finding extinction thresholds. However, interpopulation genetic variability and the subsequent potential for adaptation can be eroded before effects are noticed on species abundances. To what degree this is the case has been difficult to evaluate, partly because of the lack of both spatially extended genetic data and an appropriate framework to map and analyse such data. Here, we extend recent work on the analysis of networks of spatial genetic variation to address the robustness of these networks in the face of perturbations. We illustrate the potential of this framework using the case study of an amphibian metapopulation. Our results show that while the disappearance of some spatial sites barely changes the modular structure of the genetic network, other sites have a much stronger effect. Interestingly, these consequences can not be anticipated using topological, static measures. Mapping these networks of spatial genetic variation will allow identifying significant evolutionary units and how they vanish, merge and reorganise following perturbations.
\end{abstract}

\section{Keywords}

ecological networks, amphibian metapopulation, modularity, resilience, Alytes dickbilleni.

\section{INTRODUCTION}

Landscapes are being transformed at an unusual rate, so that populations become more and more fragmented. Metapopulation theory has described the risks of population decline and the possibility of extinction thresholds, namely values of habitat destruction at which metapopulations become extinct despite some habitat being still available (Lande 1987; Bascompte \& Solé 1996; Hanski 1998). Similarly, the emerging field of landscape genetics has described how isolation by distance and other spatially related processes shape the genetic structure of wild populations across landscapes (Manel et al. 2003; McRae \& Beier 2007; Storfer et al. 2007). Global change is expected to further alter the spatial distribution of genetic variability shown by populations inhabiting such fragmented landscapes. Assessing the rate and shape of this erosion of genetic variability is important in characterising the effects of global change on the raw material of adaptation.

In this study, we combine: (1) recent statistical methods describing how genetic variability is distributed among spatial sites, (2) network approaches to characterise the architecture of these genetic networks and (3) computer simulations addressing the effects of the sequential loss of sites.

The starting point in our perspective is the concept of population graphs (Dyer \& Nason 2004). A population graph describes the network of genetic variation among spatial sites that contains the smallest link set that sufficiently describes the patterns of genetic covariance (Dyer \& Nason 2004, see Box 1). This is different from traditional genetic approaches such as $F_{S T}$ or AMOVAs that are based on a pairwise analysis of effects, thus precluding the characterisation of the simultaneous influence of all sites. Although population graphs are certainly related to previous approaches, there

${ }^{1}$ Integrative Ecology Group, Estación Biológica de Doñana (EBD-CSIC), Américo Vespucio s/n, E-41092, Sevilla, Spain

*Correspondence: E-mail: bascompte@ebd.csic.es are subtle but important distinctions. For example, while traditional AMOVA assesses whether there is significant genetic variation, population graphs map how such a variation is distributed in space (Dyer 2007). More importantly, a network approach benefits from the tool kit of already developed analytical techniques (Urban \& Keitt 2001; Dyer 2007; Rozenfeld et al. 2008; Bascompte 2009; Fortuna et al. 2009; Dale \& Fortin 2010). Our second step is to use one of these new tools, modularity analysis, to characterise the architecture of the resulting networks of spatial genetic variation.

The concept of modularity has a long tradition in the analysis of complex networks, both in physics and sociology (Newman \& Girvan 2004; Guimerà \& Amaral 2005; Danon et al. 2006; Fortunato 2010). Modularity measures the tendency of a network to be organised in modules or compartments, where nodes within a module interact frequently among themselves, but show little interaction with nodes from other modules (see Box 2).

The search for modularity in food webs has been an active area in ecology because of the potential implications for network persistence (May 1972; Pimm \& Lawton 1980). There have been two main research directions. On one hand, ecologists have tried to find evidence for modularity in real ecological networks (Pimm \& Lawton 1980; Raffaelli \& Hall 1992; Melián \& Bascompte 2004; Olesen et al. 2007; Guimerà et al. 2010). On the other hand, researchers have explored the dynamical implications of modularity. Stouffer \& Bascompte (2011) have found that modularity increases the persistence of realistic models of food webs because it buffers the propagation of perturbations such as the extinction of a species.

More recently, modularity analysis has been applied to genetic networks both at local as well as regional scales (Fortuna et al. 2008, 2009). The rationale for focusing on modularity is twofold. First, as

'Invited manuscript for the Ecology Letters' special issue on the Ecological Effects of Environmental Change. 


\section{Box 1: Population Graphs}

Dyer \& Nason (2004) developed a network approach to study the spatial distribution of genetic variation (the so-called population graphs). The rationale for their approach is the need to move beyond the usual averaging summary statistics - as is commonly done in landscape genetics - to embrace the simultaneous effects across all sites. The main steps for calculating the network of spatial genetic variation are: (1) calculating the genetic distance between sites by translating multilocus genotypes of individuals to multivariate codification vectors and (2) estimating the conditional independence structure of the genetic covariance.

The basis of the approach of population graphs is information on spatial genetic variability. This is measured as the tendency of individual genotypes in a site to vary from each other. Each node represents a spatial site with information on the genetic variance of the individuals at this site in relation to the total variance. The procedure starts with a fully connected network in which all sites are linked to each other by their genetic similarity, which determines link strength. Thus, the squared genetic distance between two sites $i$ and $j$ can be written as (Fortuna et al. 2009):

$d_{i j}^{2}=\frac{1}{2} \sum_{k=1}^{K}\left[\frac{1}{K p_{k}}\left(y_{i k}-y_{j k}\right)^{2}\right]$,

where $y_{i k}$ is the element of the vector of the average genotype in site $i$ containing the value of allele $k$ (Smouse \& Peakal 1999); $K$ is the number of alleles; and $p_{k}$ are the allelic frequencies across sites.

The next step consists of the pruning of the above fully connected network by removing all links connecting sites whose genetic similarity is mediated by their genetic similarity with common sites. This procedure leads to a network of genetic variation containing the smallest link set that sufficiently explains the genetic covariance structure among sites. In that context, two sites will be linked if they have a significant genetic covariance after removing the covariation that each of them has with the remaining sites. To decide whether a link between sites $i$ and $j$ should be removed, one uses the following statistic (Whittaker 2004):

$\tau=-N \operatorname{Ln}\left[1-\left(r_{i j}\right)^{2}\right]$,

where $N$ is the number of individuals in the entire data set, and $r_{i j}$ is the partial correlation coefficient between sites $i$ and $j$. This statistic asymptotically follows a chi-square distribution. See Dyer \& Nason (2004) for details, and Fortuna et al. (2009) for a working example through all the steps.

stated above, modularity is clearly linked with the persistence of the network. Second, the resulting modules can be interpreted as a bottom-up classification of populations and therefore determine the relevant spatial scale. Indeed, nodes within a module are genetically much more similar than they are with nodes belonging to other modules and can therefore constitute elements of the same genetic class.

In this study, we advocate the approach of networks of spatial genetic variation in the context of global change. One preliminary step is quantifying the robustness of these networks by comparing changes in their modular structure across a gradient of habitat alteration. Here, we do so as follows. First, we perform node-removal experiments, an approach previously used to assess robustness of food webs (Solé \& Montoya 2001; Dunne et al. 2002), mutualistic networks (Memmott et al. 2004; Burgos et al. 2007; Rezende et al. 2007) and dispersal networks (Urban \& Keitt 2001). Second, we apply a recently developed method to quantify the changes in modularity through consecutive node removals (Methods). This combined approach can inform us on the rate and shape of network collapse. In the particular context of the networks of spatial genetic variation, it can provide insight, for example, on how the number of populations - and the underlying mapping of the genetic variation - will change as some local sites are being lost. Also, these simulations can be used to rank spatial sites in terms of the magnitude of the changes to the overall network following their disappearance.

We apply the above framework to genetic data of the Betic Midwife toad Alytes dickbilleni in the Sierra de Cazorla, Segura y las Villas, a mountain region in south-eastern Spain (Fig. 1). Amphibians are a good case study of spatial genetic networks. First, they are among the most endangered vertebrates on Earth (Stuart et al. 2004), showing a high sensitivity to global change (Pounds et al.
2006). Second, their patchy habitats are very amenable to be described as a network of ponds (Fortuna et al. 2006; Campbell Grant et al. 2010). As a consequence, the number of articles on landscape genetics using amphibians has steadily increased in the last few years (Arens et al. 2007; Giordano et al. 2007; Purrenhage et al. 2009; Steele et al. 2009; Murphy et al. 2010).

We illustrate the range of potential gradients of habitat alteration of the above spatial network following two contrasting scenarios. The first scenario would reflect the prevailing idea that extinction probability increases as one moves from lowlands to mountain tops - although the real pattern can be more complex than that (Pounds et al. 2006). This would be a surrogate of the expected extinction gradient due to the impact of the pathogenetic chytrid fungus (Batrachochytrium dendrobatidis), which has been reported to be most virulent at high elevations (Pounds et al. 2006) or because of negative effects of ultraviolet radiation (Lizana \& Pedraza 1998). The second scenario would be the inverse, reflecting a situation where the probability of extinction would increase from mountain tops to lowlands, as for example in the case of drought.

\section{MATERIALS AND METHODS}

\section{Field work}

Our study species is the Betic Midwife toad (Alytes dickbilleni). Its distribution is restricted to the mountains of south-eastern Spain. Adults occur usually in rocky fissures next to water sources. This amphibian has a remarkable reproductive behaviour. The male carries the eggs twined around its hind legs on land for $c$. a month, from fertilisation until they are ready to hatch (Fig. 1a). Larvae have 


\section{Box 2: Modularity}

A network is said to be modular if it tends to be arranged in groups of nodes that interact frequently among themselves, but show few interactions with nodes from other modules.

The analysis of modularity has constituted a bourgeoning field of research in the study of complex networks, with important contributions from physics and sociology. There are different methods to detect modules in complex networks, and the interested reader should refer to the thoughtful reviews by Danon et al. (2006) and Fortunato (2010).

Modularity is defined as follows (Newman \& Girvan 2004):

$M=\sum_{\text {all modules } i}$ observed fraction of edges within module $i$ - expected fraction of edges within module $i$

For the specific case of one-mode, undirected networks as the spatial networks here described, the previous expression can be written as (Newman \& Girvan 2004):

$M=\sum_{\text {all modules } i}\left(\frac{e_{i}}{L}-\sum_{\begin{array}{c}\text { all pairs of } \\ \text { species in } i\end{array}} \frac{k_{m}}{2 L} \frac{k_{n}}{2 L}\right)=\sum_{\text {all modules } i}\left(\frac{e_{i}}{L}-\frac{d_{i}}{2 L} \frac{d_{i}}{2 L}\right)$,

where $e_{i}$ is the number of edges within module $i, k_{m}\left(k_{n}\right)$ is the degree of node $m(n)$ and $d_{i}$ is the sum of the degrees of all nodes in module $i$.

Once the expression of modularity has been defined, a second challenge is to implement it in a complex network. Unfortunately, there is no exact way to do so, and one has to use some heuristic numerical approach. The idea is to try different partitions of the network in modules, to measure its modularity, and to compare it with other partitions so one ends up with the partition that maximises the previous equation. As the size of the network increases, the number of combinations become impractical to be analysed systematically. Therefore, one needs a shortcut provided by an algorithmic approach. One very successful example is simulated annealing (Guimerà \& Amaral 2005). This algorithm finds a modular structure by maximising the number of links between sites within the same module and minimising the links between sites located in different modules. This procedure is time consuming, but can handle well networks of the size of the standard ecological systems (Danon et al. 2006).

Once a significant partition of the network into modules has been found, modularity analysis also classifies each node in terms of its role in the overall topology (Guimerà \& Amaral 2005). Specifically, the importance of a node can be defined in terms of two quantities: within-module degree and participation coefficient. The former indicates how important a node is within its module in terms of its number of interactions with other nodes in that same module. The latter indicates how homogeneously distributed are a node's links are across nodes from multiple modules. The nodes with the highest participation coefficient are assumed to be very important in attaching the different modules. All this information provides from a static view, a single snapshot of the network without temporal information.
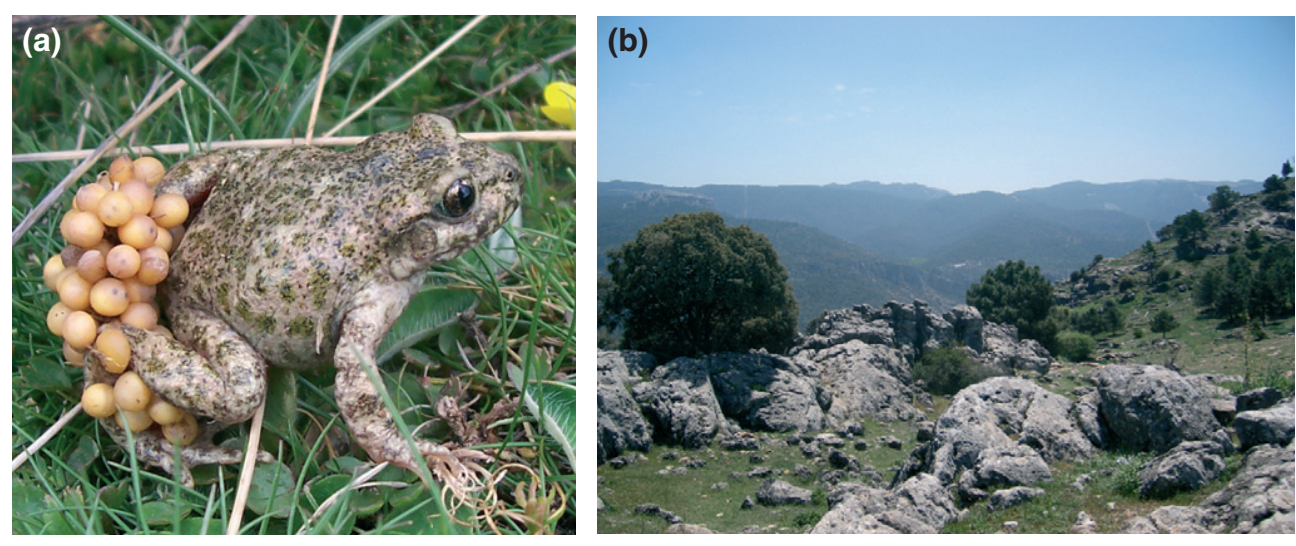

Figure 1. Our study species, the Betic Midwife toad (Alytes dickbilleni; a), is an endangered amphibian whose distribution is constrained to the mountain areas of southeastern Spain. In particular, our field site is constituted by a patchily distributed set of sites in the Cazorla mountain area (b). Pictures by Emilio González-Miras and E. M.A. respectively. 
a slow maturation rate and remain in permanent mountain streams and man-made cattle troughs. This species has become highly endangered due to the fragmentation of suitable habitats and the modernisation of agriculture.

Field sampling was conducted between 2008 and 2010 in the Natural Park of Cazorla, Segura y las Villas, Jaén Province, Spain (Fig. 1b). This is an area represented by south-eastern Spanish Mediterranean mountain vegetation on calcareous substrate. Deep soils are occupied by deciduous vegetation, while rocky exposed slopes are dominated by open pine forest and juniper (for additional details see Jordano 1995).

During the sampling period, we collected a total of 528 individual toads from 29 local sites (eight in natural sites and 21 in artificial cattle troughs). The altitudinal gradient of the different sites ranges from 414 to $1809 \mathrm{~m}$. The minimum and maximum number of individuals sampled per site was nine and 37 respectively. We sampled toe clips from adults and small tail tips $(2-5 \mathrm{~mm})$ from tadpoles. Tissue samples were stored in $70 \%$ ethanol and maintained at $-20^{\circ} \mathrm{C}$ in the lab. After tissue collection, animals were released at the site of capture.

\section{Laboratory methods}

We isolated DNA from the previously collected tissue using a standard phenol/chloroform extraction protocol (Sambrook et al. 1989). We generated multilocus genotypes using 11 polymorphic microsatellites designed for Alytes dickhilleni (Albert et al. 2011) plus two microsatellites designed for the sister species Alytes muletensis (Kraaijeveld-Smit et al. 2003). A Quiagen multiplex kit with standard conditions and a Q-solution were used to generate multiplex PCR with microsatellites of Alytes dickhilleni with $0.25 \mu \mathrm{M}$ of primer, $3 \mu \mathrm{L}$ of genomic DNA and annealing temperature of $62^{\circ} \mathrm{C}$.

Amul14 and Amul15 microsatellites were amplified, each one in a separate 'touchdown' PCR with an annealing temperature between 60 and $44^{\circ} \mathrm{C}$, and a decrease of $1^{\circ} \mathrm{C}$ at each cycle. Both microsatellites were amplified using $0.40 \mu \mathrm{M}$ dye-labelled M13 primer, $0.25 \mu \mathrm{M}$ tailed-reverse primer, $0.034 \mu \mathrm{M}$ M13 tailed-forward primer, $0.5 \mathrm{U}$ Taq DNA polymerase (Bioline) and $5 \mu \mathrm{L}$ of genomic DNA.

Amplified fragments were analysed using an ABI 3130xl Genetic Analyser with a LIZ 500 like size standard. The sizing of the genotypes was performed using GeneMapper 4.0 (Applied Biosystems). To ensure genotyping accuracy, we included a negative and a positive control per extraction and PCR, and reamplified at least 10\% of samples to screen for genotyping and human error.

\section{Network of spatial genetic variation}

We used the multilocus genotypes obtained above to build a network of genetic similarity among sites. We followed the method described by Dyer \& Nason (2004) to build and prune the network of spatial genetic variation (see Box 1). However, we took into account the observed allelic frequencies in order to calculate the matrix of genetic distances among sites, as in Fortuna et al. (2009). When these frequencies are equiprobable, the original formulation by Dyer \& Nason (2004) is recovered.

\section{Modularity analysis}

To identify non-overlapping sets of genetically similar sites (i.e., modules) we used a heuristic method based on the maximisation of a function called modularity (Newman 2004) combined with a simulated annealing optimisation approach (Guimerà \& Amaral 2005) on the network of genetic variation. Specifically, we used the weighted version of the equation in Box 2 (Guimerà et al. 2007) as it takes advantage of the strength of the links, here represented by the genetic similarity between a pair of sites. Because of the heuristic nature of the method, we ran the algorithm 100 times and used the maximum modularity value (remember this is a maximisation procedure) as the modularity of the real network.

After estimating the value of modularity, we tested to what extent this value departs significantly from random expectation. The statistical significance of modularity is calculated by performing 1000 randomisations of the network of genetic variation keeping exactly the same number of links per site, but reshuffling them randomly using a local rewiring algorithm (Gale 1957). The $P$-value was estimated as the fraction of random networks with a modularity value equal to, or higher than, the value obtained for the real network.

We also followed Guimerà \& Amaral (2005) to characterise the topological role played by each site in keeping the genetic connectivity at the landscape level (see Box 2). Specifically, for each site, we calculated its within-module degree and participation coefficient.

The comparison between the modular structure of two networks (hereafter 'change in modules') is based on the variation of information introduced by Karrer et al. (2008). The variation of information between the modular structure of two networks is the sum of the information needed to describe the modular structure of one network given the other and the information needed to describe the modular structure of the later network considering the former. Specifically, the variation of information between networks $A$ and $B$ (V $(A, B))$ is defined by:

$V(A, B)=-\sum_{x, y} P(x, y) \log \frac{P(x, y)}{P(y)}-\sum_{x, y} P(x, y) \log \frac{P(x, y)}{P(x)}$,

where $P(x)$ is the fraction of nodes assigned to module $x$ in network $A, P(y)$ is the fraction of nodes assigned to module $y$ in network $B$ and $P(x, y)$ is the fraction of nodes assigned to module $x$ in network $A$ and to module $y$ in network $B$.

The above index goes from zero (the arrangement of the nodes within modules is the same across the two networks) to $\log n$ (each node constitutes its own module in one network, and all nodes are assigned to a single module in the other network), $n$ being the number of nodes. As we were comparing networks of different sizes, we normalised this value by $1 / \log n$, and therefore our measure of change in modular organisation goes from zero to one.

Here, we applied the previous equation when comparing the original network and the subsequent one after deleting one local site. To do so, we removed the multilocus genotypes of the individuals collected in the corresponding site. Every time we removed a site, we recalculated the network of genetic variation for the remaining sites. After that, we characterised the modular structure of the resulting network and compared the resultant module composition to that of the original network of spatial genetic variation. This was done by both removing one single site and by simulating the progressive elimination of an increasing number of sites. In the latter case, we used two gradients, starting from the site at the lowest altitude and progressing towards the one at the highest altitude, and vice versa, from the site at the highest altitude to the one at the lowest altitude. 


\section{RESULTS}

The spatial network of genetic variation in Alytes dickbilleni is composed of 27 nodes and 49 links (two sites were isolated after the pruning process). This network shows a significant modular structure $(m=0.616, P<0.001)$, with $65 \%$ of the links established between nodes within the same module.

The resulting network is organised in 5 well-defined modules colour-coded in Fig. 2. There is some spatial clustering of modules, which reflects natural dispersal among the sites.

This network constituted our baseline to compare with subsequent networks once one or several local sites had been removed. First, we explored to what degree the effects of losing one local site on the modular network structure changes across sites. This provided a measure of the relative importance of each site in maintaining the architecture of the genetic network. Second, we proceeded by removing an increasing number of nodes to assess the rate and shape of the cumulative change in the network of spatial genetic variation.

As can be seen in Fig. 3a, the effect of removing one local site on the modular structure of the remaining network is quite heterogeneous. Actually, six out of the 29 sites have no effect whatsoever:

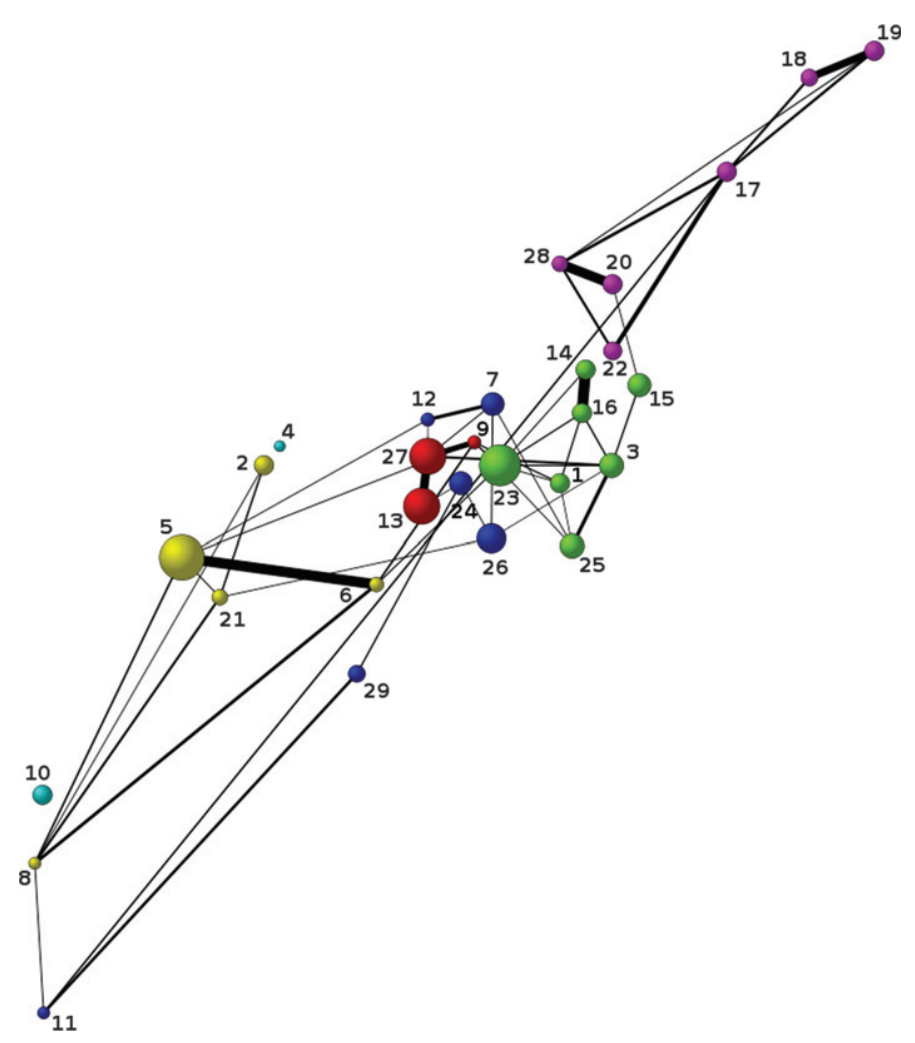

Figure 2. Observed network of spatial genetic variation in Alytes dickbilleni. In this representation, each node indicates a spatial site and each link highlights the presence of a significant genetic similarity between two such sites once the genetic similarity to other sites has been accounted for. Node position and size are proportional to its spatial coordinates in the field and to the number of sampled individuals respectively. The thickness of a link is linearly proportional to the level of genetic similarity between the two sites. Each colour indicates a module, namely a group containing genetically similar sites. This network shows a significant tendency to be organised in well-defined modules, which can be regarded as populations or management units. the resulting network has the same modular organisation as the original one in terms of the remaining nodes being allocated to the same modules than before the removal.

In contrast, other local sites have a much stronger effect, so that their removal leads to a change of c. $20 \%$ in the modular structure of the remaining network. As these changes in network structure can potentially have a large influence on dynamics (e.g. Holland \& Hastings 2008; Campbell Grant et al. 2010), it is imperative to identify these key local sites.

Given the difficulty in obtaining real data on the importance of each node to its removal, the question is to what degree these differences across local sites can be anticipated through static, topological information of the network. To answer this question, we compared the above measures of importance in terms of network robustness with the ranking of each node in terms of their participation coefficient and within-module degree, a measure of their importance at the network level and within a module respectively (see Box 2). The importance of each node regarding these two properties is represented in Fig. 3b.

Neither the participation coefficient nor the within-module degree show a significant correlation with the change in module composition once that local site has been removed $(\rho=-0.038$, $P=0.851$, and $\rho=-0.037, P=0.855$, respectively; Spearman rank correlation coefficient). Therefore, these preliminary results would suggest that none of the static indicators of the topological importance of a node can anticipate their importance in terms of robustness.

Once we have assessed the importance of the disappearance of each one of the local sites, we next explore the cumulative changes in the network of spatial genetic variation as each local site is progressively removed following our two contrasting altitudinal gradients. As noted in Fig. 4, the scenario paralleling the potential effects of UV radiation (extinction risk growing from lowlands to mountain tops) has a much greater effect on the module composition of the spatial genetic network than the contrasting scenario where extinction risk grows from mountain tops to lowlands (paralleling drought). Interestingly enough, despite the above quantitative difference, the rate of change in modular composition is similar for both scenarios. Thus, losing only a few sites has a strong effect on the genetic network and this levels off as the individuals of more sites are driven extinct (Fig. 4).

Regarding the variation in the specific number of modules - here used as surrogates of populations - this is as follows. For the lowto-high removal scenario, the number of modules remains constant until seven nodes have been removed. At this point, the number is reduced from five to four modules, a number remaining constant until 15th node is removed. From this point on, the number of modules oscillates between two and three. A similar pattern is found for the high-to-low removal scenario. Thus, the big changes in module composition observed in Fig. 4 are mainly driven by a reallocation of extant sites to different populations rather than by a fast change in the number of populations.

\section{DISCUSSION: TOWARDS A SPATIOTEMPORAL DESCRIPTION OF GENETIC VARIABILITY}

Modularity seems to be a recurrent property in networks of mating and spatial genetic variability (Fortuna et al. 2008, , 2009), although there are too few studies to assess how common this topological 

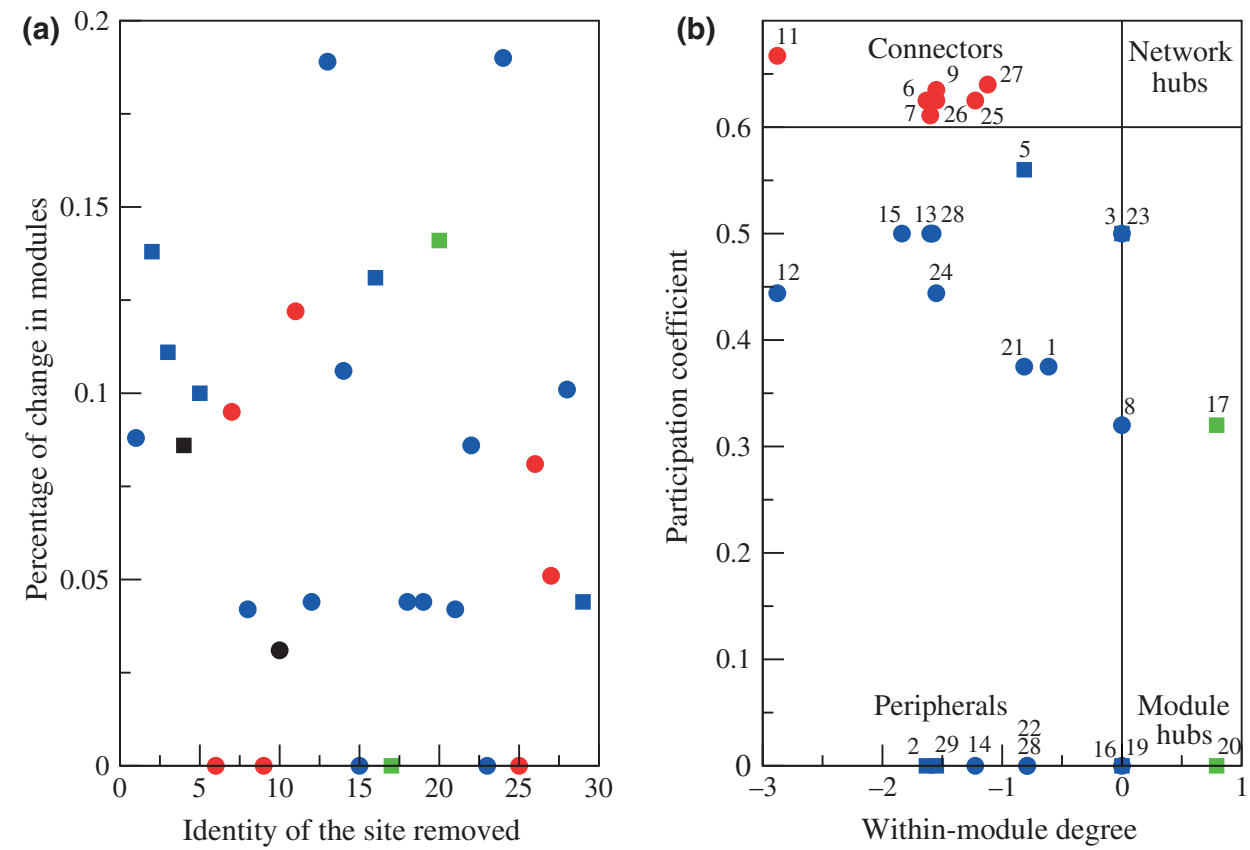

Figure 3. Importance of each spatial site at maintaining the modular architecture of the network, both dynamically (a) and topologically (b). (a) For each site removed, we calculate the modular organisation of the resulting network and compare it with that of the original network. Squares and circles indicate natural and artificial ponds respectively. The two sites in black become isolated after the pruning of the network. Other colours indicate the topological classification in panel (b). (b) Topological importance of each site according to its participation coefficient and within-module degree. A within-module degree of zero indicates that the removed node has the same number of links as the average node within that module. The vertical and horizontal lines separating the four domains are arbitrary. Numbers indicating the identity of each site are as in Fig. 2 for comparison purposes.

structure is across different species or landscapes. Thus, our first suggestion for future research would be to increase the range of species and sites where these modular analysis are performed, using the same approach to be able to compare across systems. For example, is there a phylogenetic signal in terms of the structure of these networks, with closely related species having similar architectures? What are the most important life-history traits correlating with the architecture of the networks of spatial genetic variation?

As noted earlier, modularity may constitute a more operative, bottom-up definition of a population. Therefore, the sort of network analysis advocated here can detect the spatial scale of significant evolutionary units. This is an advantage over other measures characterising the topological role of each node such as its betweens centrality which impose a particular scale - that of a node. Not surprisingly, there is no significant differences between the betweenness centrality of the nodes classified as connectors in Fig. 3b and that of the remaining 16 nodes (results not shown). Given the role of modules as an operative definition of populations, an important extension would be to explore how the number of significant evolutionary units changes as the drivers of global change increase.

The existence of a modular network has also direct implications for the differential roles of the constituent nodes and for the robustness of the overall network to perturbation. Thus, modularity implies that different nodes have very different roles in terms of keeping the overall connectivity. Nodes with the highest participation coefficient, for example, are assumed to be the most important in linking different modules. Still, our preliminary results suggest that topological information may not be enough to provide an assessment of the importance of a node in terms of changes in network structure after node removal. This makes it more difficult to predict the relative importance of the different sites, as it is much easier to rank them from the point of view of static, topological information than doing so in terms of dynamical information. This calls for an integration of the temporal dimension in spatial networks. Independently of the correlation between static and dynamic measures of a node's importance, an important extension would be to assess how the number and identity of key nodes will change with global change.

Regarding the robustness of the entire network, its modular structure can be particularly related to the propagation of fungal infection, a problem of growing concern worldwide (Stuart et al. 2004; Pounds et al. 2006; Vredenburg et al. 2010; Walker et al. 2010). Therefore, another suggestion for future work would be to consider the spatial networks as the template where the propagation of fungal infection takes place. Thus, one could deduce that, other things being equal, the propagation of fungal infections would be slowed down in a modular network in comparison with other types of networks. Actually, one recent study has found that the pattern of spread of the fungal pathogen Batrachochytrium dendrobatidis is highly influenced by the amphibian movement patterns (Vredenburg et al. 2010), which undoubtedly would affect the structure of the spatial networks of genetic variation. If global change shapes the modular structure of the spatial network, this will in turn likely affect its responsiveness to infection.

Our current results are not only very preliminary, but should be looked at as merely a way to illustrate a potentially useful approach to assessing the systemic effects of global change in the networks of spatial genetic variability. Note that here we eliminate a node and recalculate the genetic similarity across sites using the same original 


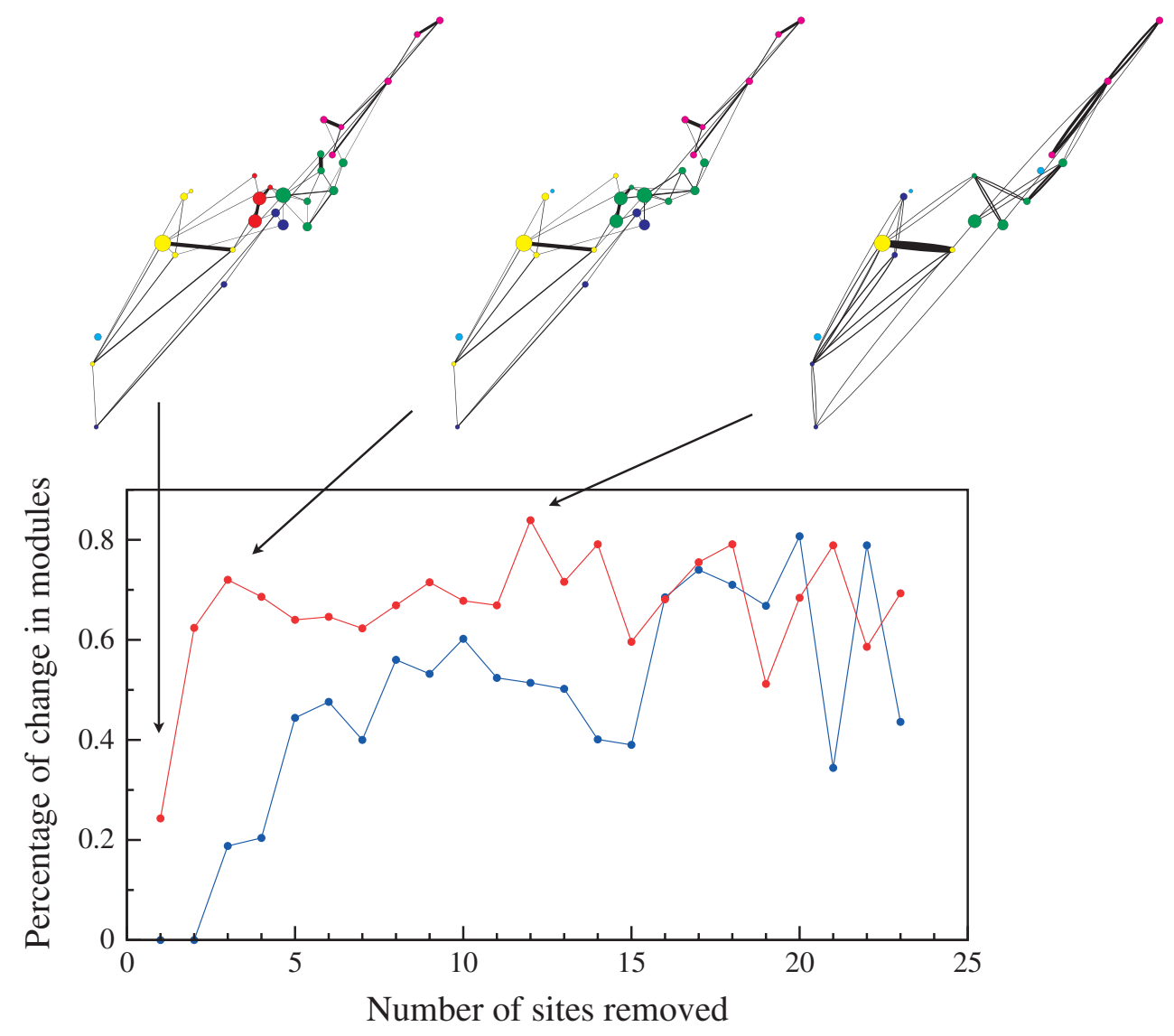

Figure 4. Cumulative effects of losing a progressively higher number of spatial sites on the modular organisation of the network. The insets represent three examples of the resulting network as in Fig. 2. Red and blue lines correspond to the contrasting scenarios of removing nodes from the highest to the lowest altitude and vice versa respectively. As in the previous figure, the change in modules measures how different the assignment of the sites is to the different modules of the perturbed network in relation to the original one when all sites were present.

data. Of course, in a real scenario, individuals may disperse to other nodes once one node has disappeared. This could affect the resulting network of genetic variability. Similarly, there may be temporal lags between habitat alteration and their effects in the network of spatial genetic variation. Future work should explore these issues with truly temporal data. Also, temporal data will serve to assess the degree to which network structure remains constant across years even when the environment is constant. For example, although a modular organisation has been postulated to potentially exert selective pressure in mating networks (Fortuna et al. 2008), the signal will be significant only if the modular structure of the network remains across time. Similarly, we need to add a functional layer on top of these networks. The most relevant achievement would be to be able to characterise the consequence of node removals on the relevant ecological processes such as gene flow or mating.

In summary, only if we can incorporate a temporal dimension to the study of networks of spatial genetic variation will we be in a position to start building a framework to assess the consequences of global change on the raw material of adaptation in heterogeneous landscapes. This not only requires temporal data, but also new tools to compare the structure of the networks through time. Hopefully, the perspective here outlined will serve to assess to what degree global change erodes the structure of these networks before demographic changes can be detected.

\section{ACKNOWLEDGEMENTS}

We thank Jaime Bosch, Marc Antoine Marchand and the staff of the Natural Park of Cazorla, Segura y las Villas for technical support and field assistance. Isabel Máximo, in turn, provided assistance at the lab. This work has been funded by a grant from the Junta de Andalucía (P07-RNM-02928). Additional funding has been provided by a Marie Curie International Outgoing Fellowship within the 7th European Community Framework Programme (to M.A.F.), and the European Research Council through an Advanced Grant (to J.B.)

\section{AUTHORSHIP}

EMA performed field work and molecular work; MAF performed network analysis; JAG supervised the molecular work; JB designed research and wrote the article with the input from all authors.

\section{REFERENCES}

Albert, E.M., Arroyo, J.M. \& Godoy, J.A. (2011). Isolation and characterization of microsatellite loci for the endangered Midwife Betic toad Alytes dickbillen (Discoglossidae). Cons. Gen. Resour., 3, 251-253.

Arens, P., van der Sluis, T., van't Westende, W., Vosean, B., Vos, C.C. \& Smulders, M.J.M. (2007). Genetic population differentiation and connectivity 
among fragmented Moor frog (Rana arvalis) populations in The Netherlands. Lands. Ecol., 22, 1489-1500.

Bascompte, J. (2009). Disentangling the web of life. Science, 325, 416-419.

Bascompte, J. \& Solé, R. (1996). Habitat fragmentation and extinction thresholds in spatially explicit models. J. Anim. Ecol., 65, 465-473.

Burgos, E., Ceva, H., Perazzo, R.P.J., Devoto, M., Medan, D., Zimmermann, M. \& Delbue, A.M. (2007). Why nestedness in mutualistic networks? J. Theor. Biol., 249, 307-313.

Campbell Grant, E.H., Nichols, J.D., Lowe, W.H. \& Fagan, W.F. (2010). Use of multiple dispersal pathways facilitates amphibian persistence in stream networks. Proc. Natl Acad. Sci. USA, 107, 6936-6940.

Dale, M.R.T. \& Fortin, M.J. (2010). From graphs to spatial graphs. Annu. Rev. Ecol. Evol. Syst., 41, 21-38.

Danon, L., Diaz-Guilera, A. \& Arenas, A. (2006). The effect of size heterogeneity on community identification in complex networks. J. Stat. Mec., P11010.

Dunne, J.A., Williams, R.J. \& Martinez, N.D. (2002). Network structure and biodiversity loss in food webs: robustness increases with connectance. Ecol. Lett., 5, 558-567.

Dyer, R.J. (2007). The evolution of genetic topologies. Theor. Pop. Biol., 71, 71-79.

Dyer, R.J. \& Nason, J.D. (2004). Population graphs: the graph theoretic shape of genetic structure. Mol. Ecol., 13, 1713-1727.

Fortuna, M.A., Albaladejo, R.G., Fernández, L., Aparicio, A. \& Bascompte, J. (2009). Networks of spatial genetic variation across species. Proc. Natl Acad. Sci. USA, 106, 19044-19049.

Fortuna, M.A., García, C., Guimarães, P.R. \& Bascompte, J. (2008). Spatial mating networks in insect-pollinated plants. Ecol. Lett., 11, 490-498.

Fortuna, M.A., Gómez-Rodríguez, C. \& Bascompte, J. (2006). Spatial network structure and amphibian persistence in stochastic environments. Proc. $R$. Soc. Lond. B, 273, 1429-1434.

Fortunato, S. (2010). Community detection in graphs. Phys. Rep., 486, 75-174.

Gale, D. (1957). A theorem of flows in networks. Pacific J. Math., 7, 1073-1082.

Giordano, A.R., Ridenhour, B.J. \& Storfer, A. (2007). The influence of altitude and topography on genetic structure in the long-toed salamander (Ambystoma macrodactulym). Mol. Ecol., 16, 1625-1637.

Guimerà, R. \& Amaral, L.A.N. (2005). Functional cartography of complex metabolic networks. Nature, 433, 895-900.

Guimerà, R., Sales-Pardo, M. \& Amaral, L.A.N. (2007). Module identification in bipartite and directed networks. Phys. Rev. E., 76, 036102.

Guimerà, R., Stouffer, D.B., Sales-Pardo, M., Leicht, E.A., Newman, M.E.J. \& Amaral, L.A.N. (2010). Origin of compartmentalization in food webs. Ecology, 91, 2941-2951.

Hanski, I. (1998). Metapopulation dynamics. Nature, 396, 41-49.

Holland, M.D. \& Hastings, A. (2008). Strong effect of dispersal network structure on ecological dynamics. Nature, 456, 792-794.

Jordano, P. (1995). Frugivore-mediated selection on fruit and seed size: birds and St. Lucies cherry. Prunus mabaleb. Ecology, 76, 2627-2639.

Karrer, B., Levina, E. \& Newman, M.E.J. (2008). Robustness of community structure in networks. Phys. Rev. E, 77, 046119.

Kraaijeveld-Smit, F.J.L., Rowe, G., Beebee, T.J.C. \& Griffiths, R.A. (2003). Microsatellite markers for the Mallorcan midwife toad Alytes muletensis. Mol. Ecol. Notes, 3, 152-154.

Lande, R. (1987). Extinction thresholds in demographic models of territorial populations. Am. Nat., 130, 624-635.

Lizana, M. \& Pedraza, E.M. (1998). The effects of UV-B radiation on toad mortality in mountainous area of central Spain. Cons. Biol., 12, 703-707.

Manel, S., Schwartz, M.K., Luikart, G. \& Taberlet, P. (2003). Landscape genetics: combinig landscape ecology and population genetics. Trends Ecol. Evol, 18, 189-197.

May, R.M. (1972). Will a large complex system be stable? Nature, 238, 413-414.

McRae, B.H. \& Beier, P. (2007). Circuit theory predicts gene flow in plant and animal populations. Proc. Natl. Acad. Sci. USA, 104, 19885-19890.

Melián, C.J. \& Bascompte, J. (2004). Food web cohesion. Ecology, 85, 352-358.

Memmott, J., Waser, N.M. \& Price, M.V. (2004). Tolerance of pollination networks to species extinctions. Proc. Biol. Sci., 271, 2605-2611.
Murphy, M.A., Dezzani, R., Pilliod, D.S. \& Storfer, A. (2010). Landscape genetics of high mountain frog metapopulations. Mol. Ecol., 19, 3634-3649.

Newman, M.E.J. (2004). Fast algorithm for detecting community structure in networks. Phy. Rev. E, 69, 1-5.

Newman, M.E.J. \& Girvan, M. (2004). Finding and evaluating community structure in networks. Phys. Rev. E, 69, 026113

Olesen, J.M., Bascompte, J. \& Jordano, P. (2007). The modularity of pollination networks. Proc. Natl Acad. Sci. USA, 104, 19891-19896.

Pimm, S.L. \& Lawton, J.H. (1980). Are food webs divided into compartments? J. Anim. Ecol., 49, 879-898.

Pounds, J.A., Bustamante, M.R., Coloma, L.A., Consuegra, J.A., Fogden, M.P.L., Foster, P.N., La Marca, E., Masters, K.L., Merino-Viteri, A., Puschendorf, R., Ron, S.R., Sanchez-Azofeifa, G.A., Still, C.J. \& Young, B.E. (2006). Widespread amphibian extinctions from epidemic disease driven by global warming. Nature, 439, 161-167.

Purrenhage, J.L., Niewiarowski, P.H. \& Moore, F.B.G. (2009). Population structure of spotted salamanders (Ambystoma maculatum) in a fragmented landscape. Mol. Ecol, 18, 235-247.

Raffaelli, D. \& Hall, S. (1992). Compartments and predation in an estuarine food web. J. Anim. Ecol, 61, 551-560.

Rezende, E.L., Lavabre, J.E., Guimarães, P.R., Jordano, P. \& Bascompte, J. (2007). Non-random coextinctions in phylogenetically structured mutualistic networks. Nature, 448, 925-928.

Rozenfeld, A.F., Arnaud-Haond, S., Hernández-García, E., Eguíluz, V.M., Serrão, E.A. \& Duarte, C.M. (2008). Network analysis identifies weak and strong links in a metapopulation system. Proc. Natl Acad. Sci. USA, 105, 18824-18829.

Sambrook, J., Fritsch, E.F. \& Maniatis, T. (1989). Molecular Cloning: A Laboratory Manual, 2nd edn. Cold Spring Harbor Laboratory Press, NY, Cold Spring Harbor, USA, p. 1659.

Smouse, P.E. \& Peakal, R. (1999). Spatial autocorrelation analysis of individual multiallele and multilocus genetic structure. Heredity, 82, 561-573.

Solé, R. \& Montoya, J.M. (2001). Complexity and fragility in ecological networks. Proc. R. Soc. B. Biol. Sci., 268, 2039-2045.

Steele, C.A., Baumsteiger, J. \& Storfer, A. (2009). Influence of life-history variation on the genetic structure of two sympatric salamander taxa. Mol. Ecol., $18,1629-1639$.

Storfer, A., Murphy, M.A., Evans, J.S., Goldberg, C.S., Robinson, S., Spear, S.F., Dezzani, D.E., Vierling, L. \& Waits, L.P. (2007). Putting the 'landscapes' in the landscape genetics. Heredity, 98, 128-142.

Stouffer, D.B. \& Bascompte, J. (2011). Compartmentalization increases food-web persistence. Proc. Natl. Acad. Sci. USA, 108, 3648-3652.

Stuart, S.N., Chanson, J.S., Cox, N.A., Young, B.E., Rodrigues, A.S.L., Fischman, D.L. \& Waller, R.W. (2004). Status and trends of amphibian declines and extinctions worldwide. Science, 306, 1783-1786.

Urban, D. \& Keitt, T. (2001). Landscape connectivity: a graph-theoretic perspective. Ecology, 82, 1205-1218.

Vredenburg, V.T., Knapp, R.A., Tunstall, T.S. \& Briggs, C.J. (2010). Dynamics of an emerging disease drive large-scale amphibian population extinctions. Proc. Natl Acad. Sci. USA, 107, 9689-9694.

Walker, S.F., Bosch, J., Gomez, V., Garner, T.W.J., Cunningham, A.A., Schmeller, D.S., Ninyerola, M., Henk, D.A., Ginestet, C., Arthur, C.P. \& Fisher, M.C. (2010). Factors driving pathogenicity versus prevalence of amphibian panzootic chytridiomycosis in Iberia. Ecol. Lett., 13, 372-382.

Whittaker, J. (2004). Graphical Methods in Applied Multivariate Statistics. John Wiley and Sons, New York. 